Gazi University
Journal of Science
PART C: DESIGN AND TECHNOLOGY
http://dergipark.gov.tr/gujsc

\title{
Student Modelling on Language Teaching Based on Bayesian Networks
}

\author{
Selçuk ŞENER ${ }^{1, *}$ (D)Ali GÜNEŞ² \\ ${ }^{I}$ Istanbul Aydin University, School of Foreign Languages,34290, Küçükçekmece/ISTANBUL \\ ${ }^{2}$ Istanbul Aydin University Facultiy of Engineering, Department of Computer Engineering,34290, Küçükçekmece/ISTANBUL
}

\section{Article Info \\ Research article Received:28/04/2021 Revision:18/05/2021 \\ Accepted:19/05/2021 \\ Keywords \\ Bayesian Networks Influence Diagrams Intelligent Tutoring Systems \\ StudentModelling}

\begin{abstract}
Acquiring a reliable student model is the principal task of an Intelligent Tutoring System (ITS). A precisely defined student model is also the key term for the success of ITSs. In this paper, a study on inferring the accurate student model on language learning is offered by utilizing artificial intelligence. In addition to the general structure of an ITS, a probabilistic model for inference using Bayesian Networks is stated in the paper. Bayesian Networks are acyclic directed graphs in which nodes represent random variables and arcs represent direct probabilistic dependences among them. In this study, graphical models and structures are implemented in a general-purpose decision modelling system SMILE and its Windows user interface, GeNIe, developed at the Decision Systems Laboratory. GeNIe user interface is also used in this study to perform Influence diagrams. Influence diagrams represent decision problems and help to choose a decision alternative with the highest expected gain. Toward the end of the study, an ITS student model which is directly associated with a standard proficiency level is aimed to be developed. This model is also complemented with a domain model, which incorporates language components such as grammar, vocabulary, and functions of language in different cases. At the end of the study, an evaluation of the model is performed with an experimental study and the results show that the participants worked with offered ITS model gathered close results when compared to those obtained by the participants who worked with a real tutor.
\end{abstract}

\section{INTRODUCTION}

Intelligent tutoring systems (ITS) can be seen as an important step forward for real adaptive learning environments. With the help of ITSs, it is aimed to achieve an adaptive, personalized approach to learning. ITSs are defined as educational systems, which utilize artificial intelligence in computer software to assist learning process [1]. They are constructed on cognitive psychology as the underlying theory of learning and deals mainly with problems such as the interpretation and structure of information within human memory as well as the nature of human error [1].

With the help of computer aided learning systems, it is aimed to provide students with an adaptive curriculum through programmed instructions. Use of computer systems in education started with a project called Plato which is developed at University of Illinois in 1950 [2]. In the following years, several computer-aided systems have been developed and these systems helped students improve their learning performances. The students gained more control over different learning stages with the help of successful multimedia technologies [3]. However, early computer aided systems couldnot go beyond providing standard education environments, although they are generally designed according to the responses obtained from students. Many computer-aided programs can only provide a usual set of instructions for different student models without evaluating student performance [4]. 
ITSs can be seen as educational software incorporating components, which usually harness AI. One of the main purposes of ITSs is to track user competence on a specific subject. Gathering information about student is a key point to make inferences about student's ability. This leads to successful and tailor-made tutoring experiences for ITSs. It can be simply defined that the basic underlying idea of ITSs is to realize that each student is unique [5]. Specialized instruction tutoring with respect to student's existing level can easily accomplish this underlying idea.

In recent years, the use of ITSs on several complex domains has become widespread. ITS applications can be encountered on a wide variety of subjects and different domains. Algebra Tutor PAT is an important and a successful example, which aims to provide tutoring for introductory algebra [6]. Another example, Auto Tutor is developed to assist students in learning computer hardware and operating systems by simulating the discourse patterns and pedagogical strategies of a human tutor [7]. Cardiac Tutor which, aims to support advanced cardiac support techniques to medical personnel, is designed to simulate various cardiac problems and students are required to select appropriate medical responses [8]. Andes is also another tutoring system, which is designed to assist students about introductory physics course. Andes tutoring systems help students to solve physics problems by providing pedagogically useful guidance.

One of the common problems of creating successful ITSs is building a structure, which fully corresponds to the field it is built on. It is generally a hard subject to address various demands of tutoring for different learning environments. Language learning is one those fields which is full of restrictive and unrestrictive domain uncertainties for reading, writing, speaking, and listening and those aspects may occur on different occasions and different context. ITSs that have long been created are generally used for learning fields with a linear syllabus with concrete learning points and those used for language learning were mainly used for only one aspect of language learning such as grammar or vocabulary. Another problem for ITSs for language learning is that they do not refer to a certain language proficiency levels or they do not clearly address the targeted student specifications as children or adults. This is where the distinctness of the model created in this paper comes into prominence.

This paper aims to outline the general structure of ITSs in computer assisted language learning. The focus of the paper is on the development and functions of student model, which is one of the four main components of conventional intelligent tutoring systems. Student model presented in this study is also complemented with Bayesian Network that utilizes probabilistic graphical models and Bayesian inference. Since student modelling includes a certain amount of uncertainty, a Bayesian Network, which is based on probabilistic theory, is an important option to overcome the uncertainty [9]. Several Bayesian Student Models have been developed in various educational applications [10]. Bayesian Networks have also been developed for several uncertain problems including NASA's space shuttle propulsion systems and as an Office Assistant in the Microsoft Windows operating system [9].

\section{INTELLIGENT TUTORING SYSTEMS FOR LANGUAGE LEARNING}

It is obvious that the use of audio-visual devices and especially computers in different phases of education generally provides substantial results. When compared different fields of education, natural language learning can be seen as a complicated subject since it both requires knowledge and skill-based approach [11]. However, learning process of a student can be improved with the help of a computer tutor and the computer tutor with in ITSs can determine the syllabus, provide guidance, and it allows the student to experience a learning process in his or her own pace.

Several ITSs for language learning have been developed regarding various languages. These systems have been constructed utilizing different structures with different pedagogical models. While some of them providing a learning environment, which is only composed of vocabulary, some others concentrated on grammar only. Since language learning itself is a complicated subject with various knowledge and skill bases, tutoring systems developed on behalf of language learning are also complicated in terms of structure and application. 


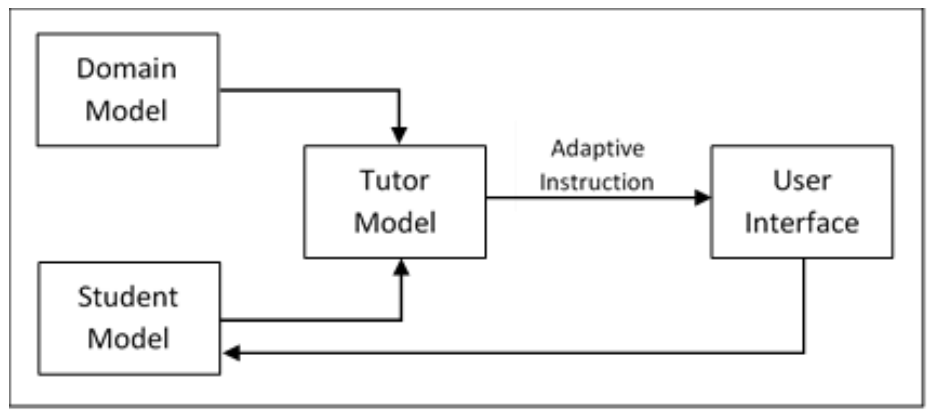

Figure 1. General Structure of an ITS.

Although there is no consensus on how an ITS for language learning must be constructed, they are generally constructed with four basic modules: domain module, student module, tutor module and user interface module, as seen in Figure 1 [4][12]. The student module is used to create a model, which reflects the current knowledge state of the student. This module usually makes use of artificial intelligence to get a precise description of the knowledge state. Domain model, which is also referred to as expert model, represents the knowledge and rules that student needs to acquire in the field of education. The next module, tutoring module is responsible to provide guidance and feedback to the student by generating different types of activities suitable for the field of study. User interface module is the last module, which interacts with the student using a graphical user interface.

Adaptation and use of computers and other technologies in language learning dates to 1960. Providing individualized guidance for vocabulary and grammar studies was the main concern of early ITSs for language learning. Natural language learning is a complicated issue which incorporates the development of both receptive (listening, reading) and productive (speaking, writing) skills. However, a frequently noticed issue in ITSs for language learning is over-restricting the learning domain [13]. A general tendency within ITSs is to reside in a single linguistic skill, instead of dealing with each individual skill $[4][13]$.

The assessment of certain linguistic skills and functions (e.g., reading, listening and grammar) can be standardized and individually carried out by intelligent tutors. However, some skills are especially difficult for the machine to be evaluated by the machine [13]. It is true that computers cannot have the ability to fully evaluate unrestricted productive skills, namely speaking and writing, but ITSs may find various ways to accomplish such ability within a restricted domain. In addition, with recent technological advancements computers and their peripheral devices are not the same as they are in the past.

\section{STUDENT MODELLING FOR AN INTELLIGENT TUTORING SYSTEM}

Student modelling for ITSs is generally considered to be atough subject. However, the success of an ITS is directly related with how student modelling has been developed. A student model in an intelligent tutor observes student behavior and creates a qualitative representation of her cognitive and affective knowledge [4]. A successfully gathered student model which directly reflects the current knowledge state of its user will be a valuable input for other components of the ITS. In this study, a student modelling component of an ITS for English Language Learning is developed.

One of the issues for language teaching ITSs is the inability of an ITS to provide students with different levels of language proficiency (e.g., Common European Framework of Reference for Languages (CEFR) proficiency levels A1-C2) [13]. This inability leads them to be deemphasized and move away from being a standard education platform in terms of language learning. Keeping this in mind, the student model developed in this study is intended to classify student's knowledge state regarding CEFR proficiency level A1. Domain model of in this study is designed to comply with the standards of CEFR level A1. Topic, grammar, vocabulary and other materials for English language are selected in accordance with 'Can Do Descriptors' defined by CEFR [14]. 
The student model handled in this paper does not only observe the student's English grammar knowledge level, but also presents a suitable vocabulary checker with respect to A1 proficiency. In addition, a function component which reflects the student's use of English in different cases (e.g., greetings, responding to a phone call etc.) is also included.

Student modelling in ITS is an important subject which is generally full of uncertainty [4] [15]. Obtaining a knowledge state of a student upon a particular topic is not a thing, which can be resolved using a simple logic. To gather information about a specific topic, sometimes it is necessary to get knowledge levels of related or prerequisite topics as well. Moreover,it is possible that a student may answer a question correctly by a mere chance, which may lead to a wrong observation and eventually an erroneous inference. Bayesian Networks are regarded as a reliable option to overcome this uncertainty and reduce complexity [15] [16].

\subsection{Bayesian Networks In Its}

Bayesian networks are graphical models designed to explicitly represent conditional independence among random variables of interest. Bayesian networks in ITSs make use of this information to decrease the complexity of probabilistic inference [17]. Language learning is a field full of correlated subject and unrestricted domain [13]. Although there are some other options to be used in ITSs such as Hidden Markov, Reinforcement Learning, Bayesian Networks are considered as a reliable solution to solve uncertainty in ITSs. Intelligent tutors use BBNs to aid classification and forecasting for tutoring, design student awareness, forecast student behavior, make tutoring decisions, and (combined with student mastery data) decide which actions students will need assistance and their likely method of solving problems [4] [9] [18].

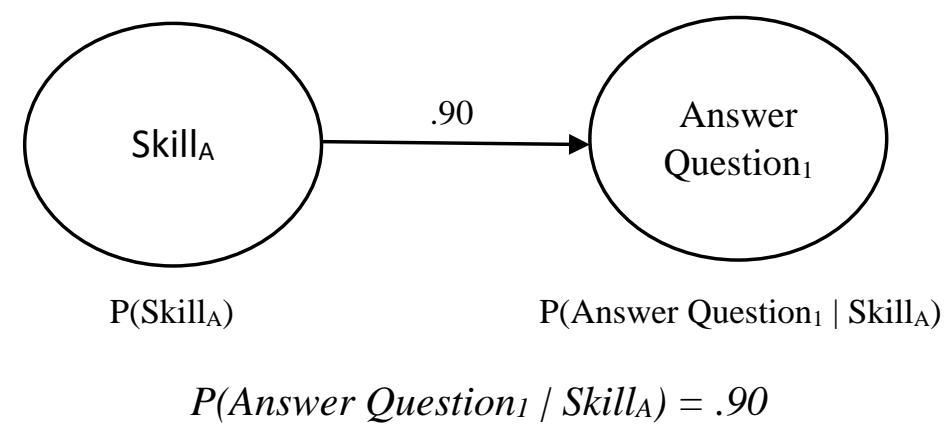

Figure 2. Graphical Network for a basic propositional belief [4].

In Figure 2, there is a close relationship between SkillA and Answer for Question1. It can also be inferred that students with SkillA are likely to have a chance to answer Question1 correctly. In order to model student knowledge in ITSs, several Bayesian Network models have been developed so far. Pump Algebra Tutor, which models algebra problem solving, Animal Watch, which provides tutoring for mathematics skill, and The Cardiac Tutor, which helps student learn an established medical procedure, utilize Bayesian Networks [4]. According to Mayo and Mitrovic, those Bayesian Networks can be classified under 3 main categories as expert-centric, data-centric, and efficiency-centric [18].

\section{METHOD USED FOR THE ITS MODEL DEVELOPED IN THE STUDY}

In this study, expert-centric model is used to define a suitable Bayesian Network to model student knowledge on English language. Expert-centric methods are utilized in several early tutors [4]. Andes, which used a basic Bayesian Network to infer a student's knowledgeis based on performance[19]. Another example, HYDRIVE that is used to give instructions for problem solving and troubleshooting in flight line mechanics also uses an expert-centric student model [20]. In expert-centric Bayesian Network 
Models, general network structure and the topology are defined by experts and for the student model presented in this paper, expert teachers of English language defined the structure, node relations and conditional probabilities.

\subsection{Components Of The Model}

When creating the structure and the topology of Bayesian model,the focus was on selecting a curriculum in compliance with CEFR proficiency levels for English language. The curriculum of the study is designed regarding A1 level for English language. Considering most ITSs for language learning lacks this feature, it was highly important to design the syllabus in accordance with a standard level from CEFR.

In this paper, only a part of the curriculum is presented to give an idea about the general structure of Student Modelling. In Figure 3, basic structure of this model can be seen in detail. The model shown in Figure 3 was developed using GeNIe Software. GeNIe is an interactive model building and exploration tool. It is used to construct Bayesian Networks, dynamic Bayesian Networks, and influence diagrams.

Model in Figure 3 mainly includes two types of nodes as evidence and decision nodes. It illustrates that, when necessary evidence obtained, required inferences can be completed for Topic 1. Topic 1 is complemented with three subtopics, Grammar, Functions and Vocabulary. Between Topic 1 and the subtopics, arcs can be observed going from subtopics to Topic 1, which indicates that conditional probabilities are set accordingly.

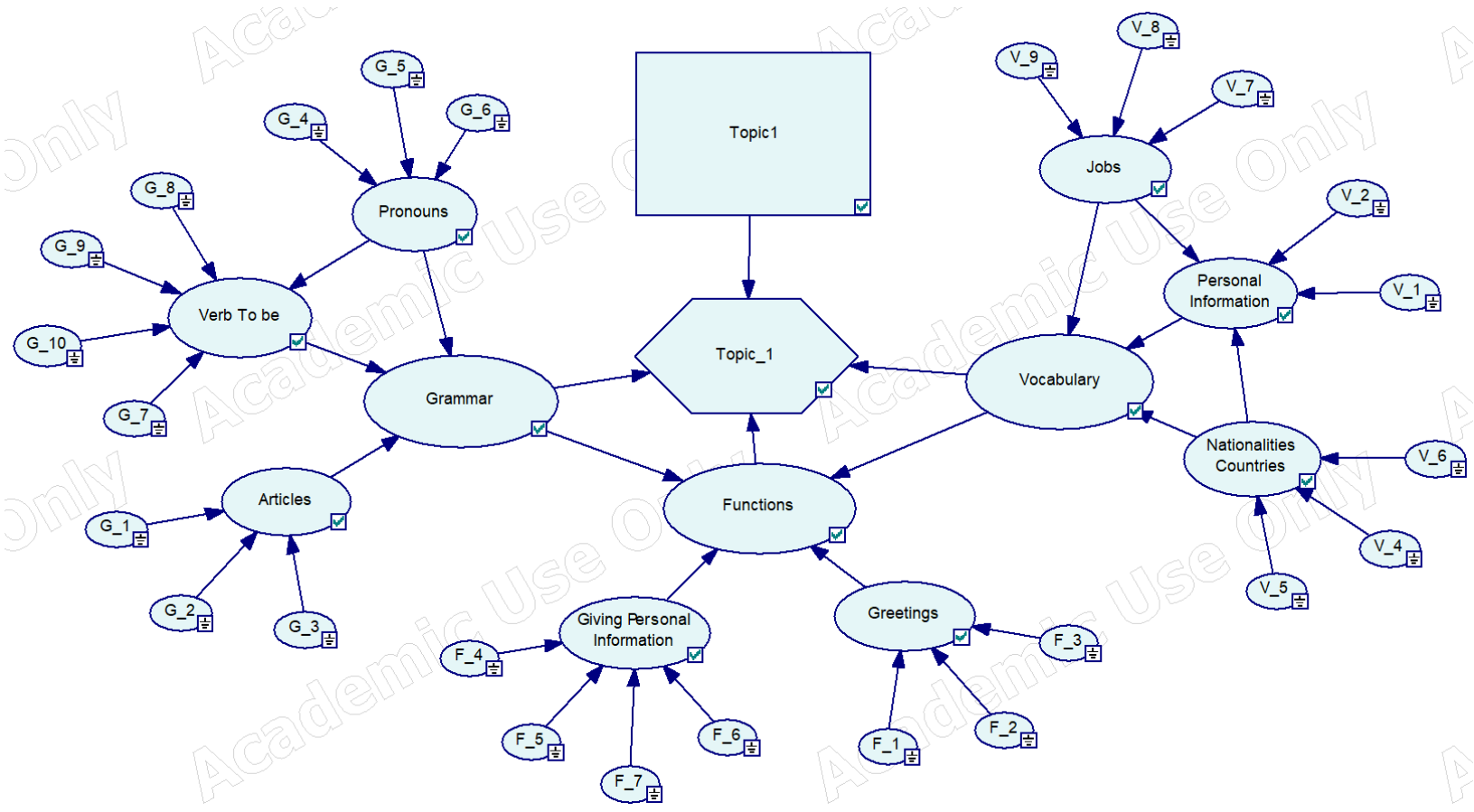

Figure 3. Basic Structure of the Model in this study.

Similar to the relation between Topic1 and subtopics, it can also be observed that granularity increases by going down on the topology. As seen in Figure 3, Grammar subtopic has also child nodes defining specific grammar topics with observable, in other words, evidence nodes under them. Those evidence nodes are created to get student involved through questions, exercise and gather information for knowledge state.

For Grammar subtopic, three child nodes can be observed as Articles, Pronouns and Verb to be. Those grammar subjects are selected in accordance with A1 level of English language. For each child node, there are three observable nodes. Those nodes have two states as correct and wrong. It can also be observed that there is an arc going from Pronouns to Verb to be which defines that the subject Verb to be 
is conditionally dependent on Pronouns, since precise skill on Verb to be can only be possible with a good command on pronouns. In Figure 4, conditional probability distribution for this subtopic can be seen.

\begin{tabular}{|c|c|c|c|c|c|c|c|c|c|}
\hline \multirow{2}{*}{\multicolumn{2}{|c|}{$\begin{array}{c}\text { Articles } \\
\text { Pronouns }\end{array}$}} & \multicolumn{4}{|c|}{ Known } & \multicolumn{4}{|c|}{ Not_Known } \\
\hline & & \multicolumn{2}{|c|}{ Known } & \multicolumn{2}{|c|}{ Not_Known } & \multicolumn{2}{|c|}{ Known } & \multicolumn{2}{|c|}{ Not_Known } \\
\hline & Verb To be & Known & Not_Known & Known & Not_Known & Known & Not_Known & Known & Not_Known \\
\hline$\nabla$ & Known & 0.99 & 0.66 & 0.66 & 0.33 & 0.66 & 0.33 & 0.33 & 0.01 \\
\hline & Not_Known & 0.01 & 0.34 & 0.34 & 0.67 & 0.34 & 0.67 & 0.67 & 0.99 \\
\hline
\end{tabular}

Figure 4. Grammar Subtopic Conditional Probability Table

Other subtopics depicted in Figure 3, Function and Vocabulary nodes are designed, respectively. GeNIe software has two types of views as Icon and Bar Chart. As shown in Figure 3 nodes can be seen in Icon view and arcs showing the relationship between nodes are clearly depicted. However, in Bar Chart view, initial states of the nodes and their probabilistic outcome can be observed.

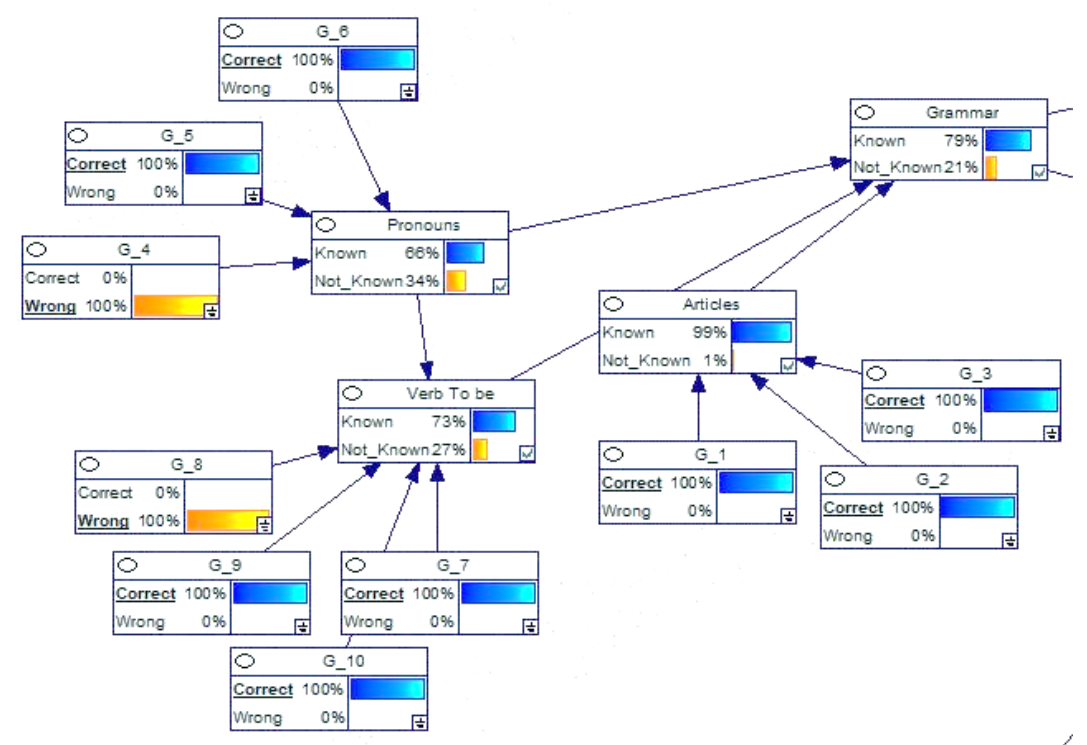

Figure 5 Updated Grammar Subtopic

When the model updated by observed evidence coming from lowermost nodes, it becomes possible for the system to make an inference about the knowledge state of the student. Figure 5 shows the updated view of the subtopic Grammar and for Grammar subtopic, Pronouns has two correct answers from its child nodes, 'Verb to be' has three out of four and Articles has three out of three. Consequently, with these results from childnodes, Grammar subtopic has a probability percentage of 79\% for 'Known' state, which proves that grammar knowledge is around an acceptable known state for Topic 1.

Influence diagrams (IDs) introduced by [21], are acyclic directed graphs modelling decision problems under uncertainty. An ID encodes three basic elements of a decision: (1) available decision options, (2) factors that are relevant to the decision, including how they interact among each other and how the decisions will impact them, and finally, (3) the decision maker's preferences over the possible outcomes of the decision-making process[22]. 


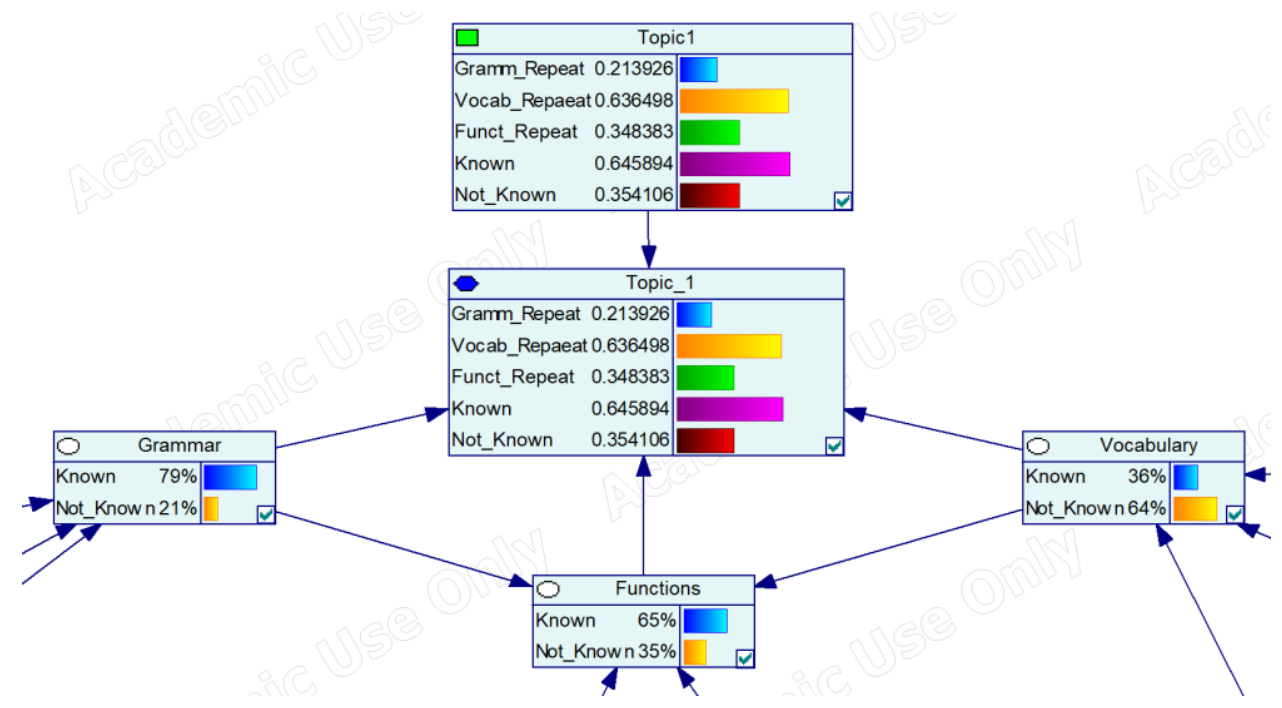

Figure 6.Detailed View of Topic 1 and Subtopics.

As seen in Figure 6, a decision node and a value node are used to possess an inference for the knowledge level of Topic 1. Figure 6 depicts a detailed view of decision node for Topic 1 and three subtopics respectively with updated states. Grammar is supposed to be known by $79 \%$ and Functions with $65 \%$. However, it is observed that Vocabulary result is 'Not_Known' by $64 \%$. Accordingly, within the decision node on top, probability of Topic 1 to be 'Known' is 0.645 , although one of the subtopics is observed as 'Not_Known'. However, the decision node states an inference that Vocabulary subtopic must be repeated, with a high probability of 0.636 .

\section{APPLICATION OF THE ITS MODEL}

In ITSs, student modelling is used to provide the necessary input for adaptive guidance to the system. Accordingly, the success of student modelling within an ITS directly effects the achievement of the guidance. With the help of student knowledge state, which is obtained through a Bayesian Network, ITSs can provide adaptive, individual guidance for the users. To test the accuracy of the student modelling defined in this study, a tutoring module based on generating learning sequence has been implemented.

\subsection{Tutor Module}

Tutoring module in ITSs, includes a set of rules and procedures belonging to the subject to be taught. Those rules are used to define how the system should respond to its user by utilizing the data coming from the student model [23]. Tutoring module implemented for this study is based on generating learning sequence and it provides adaptive guidance for three main topics. One of those topics is defined in student modelling phase of the study. According to the output from student modelling module, tutor module may provide remedial, move to the next level in the curriculum or force the user to repeat the subject that is not successfully completed.

\subsection{User Interface Module}

The user interface module works as a means to interact with the user. The user interface keeps the interaction between the user and the system, and the success of the system is closely related to this module no matter how the internal system is intelligent. In order to accompany other modules defined in this study, a user interface module has been created. This user interface module includes multiple choice questions, gap filling and vocabulary drag-drop exercises according to the topic. The user interface model is used to update knowledge state in student modelling module, and it also provides an effective presentation of the curriculum directed tutor module of the system. 


\section{RESULTS AND CONCLUSION}

Accuracy and correctness of the student modelling and accordingly the effectiveness of tutoring provided by the system is obviously important. To evaluate the accuracy of the student-modelling component of the system that is defined in this study, an experiment with 120 participants carried out. Participant are divided into three groups. The first group consisting 40 participants used the system with adaptive tutoring, second group of 40 participants just worked alone without any tutoring over an instruction book which also includes exercises on the subjects. Final group of 40 participants studied accompanied by a teacher with instruction books provided. The experiment took 3 days and 90 minutes each day. The curriculum covered in the experiment covered three main subjects and three secondary subjects, which are similar to the ones defined in student modelling part of the study. All the participants were high school students, and they were supposed to have the same English proficiency levels.

At the end of the experiment, participants took a test over three subjects including 75 questions, 25 questions for each subject. The overall result of the test given to the participants can be seen in Table 1 .

Table 1. Overall Success Average Values

\begin{tabular}{|l|c|c|c|}
\hline \multirow{2}{*}{} & \multicolumn{3}{|c|}{ Overall Success Average Values } \\
\cline { 2 - 4 } & Subject 1 & Subject 2 & Subject 3 \\
\hline Control Group Studied Alone & $54 \%$ & $62 \%$ & $58 \%$ \\
\hline Control Group Studied with Teacher & $73 \%$ & $80 \%$ & $76 \%$ \\
\hline Experimental Group Using ITS & $68 \%$ & $79 \%$ & $72 \%$ \\
\hline
\end{tabular}

It can be observed from Figure 7 that the participants studied over ITS obtained close results for all three subjects when compared to that of participants studied tutored by a teacher in classroom environment. The participants who worked alone achieved relatively low results. Figure 8 also depicts that the average final scores for experimental group studied with the ITS gathered close results to that of control group studied with a teacher.

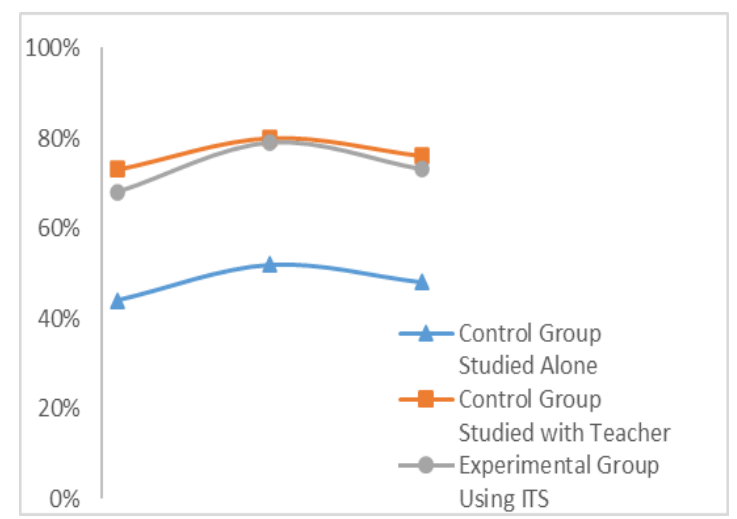

Figure 7. Overall Success over 3 Subjects

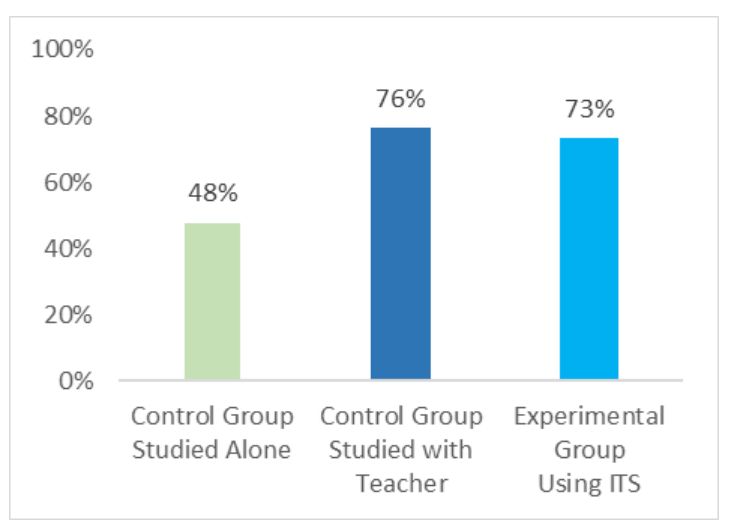

Figure 8. Average Scores

The idea of using computers in education, especially in language learning has much changed since it was first uttered. With a boost in technology, Intelligent Tutoring Systems utilizing Artificial Intelligence techniques have also much changed since they were first developed. Although language learning is a complex field with an unrestricted domain, ITSs can still be developed to replace human tutors, and provide individualized guidance for the students with their own pace. 
In this paper, it is aimed to define a beneficial student modelling structure for language teaching ITSs. It is obvious that only with the help of an effective student modelling component, a tailor-made, individualized tutoring, and guidance can be possible. Since language learning has an unrestricted domain most of the time, it is important to develop a tutoring structure with respect to a set of standards upon which the majority agreed such as CEFR proficiency levels. That is why the use of similar standards in the Bayesian Network Module is emphasized. Another key point for the success of ITSs in language learning is to create high granularity of the content on both student modelling and tutoring domain as well. Since student modelling for language tutors is full of uncertainty, it is extremely beneficial to provide high granularity.

One way of evaluating ITSs to be successful is to compare the results obtained with the learning outcomes obtained with a human tutor. A major idea behind creating intelligent tutors is to provide adaptive, individual learning environment and the main concern is that whether replacing human tutors is possible or beneficial as well. The experiment implemented in this study proves that when an accurate student modelling is established with effective tutoring model, it is possible to obtain remarkable results. However, the difficulty and complexity of creating such systems should not be underestimated and required steps to establish such systems should be taken accordingly.

As a future work, equipping an ITS with appropriate student modelling and tutoring structures that can respond to both restrictive and unrestrictive domain of language learning will be the focus. Also developing an ITS for language learning which completely fulfil the tutoring needs of both productive and receptive skills will be another subject to be focused on.

\section{REFERENCES}

[1] V. J. Shute and J. Psotka, "Intelligent Tutoring Systems: Past, Present, and Future." US Dept of the Air Force, Tech. Rep., May 1994.

[2] Molnar, "Computers in Education: A Historical Perspective of the Unfinished Task," T H E Journal (Technological Horizons in Education), vol. 18, no. 4, p. 80, 1990.

[3] J. Kosakowski, “The Benefits of Information Technology. ERIC Digest., 1998-Jun,” Tech. Rep., Jun 1998.

[4] B. P. Woolf, Building Intelligent Interactive Tutors. Elsevier, 2009, pp. 60-94.

[5] R. Santhi, B. Priya, and J. M. Nandhini, "Review of Intelligent Tutoring Systems Using Bayesian Approach," Feb 2013.

[6] S. Ritter, J. Anderson, M. Cytrynowicz, and O. Medvedeva, "Authoring Content in the PAT Algebra Tutor," Journal of Interactive Media in Education, vol. 1998, no. 2, p. 9, oct 1998.

[7] A.C.Graesser, K. Wiemer-Hastings, P. Wiemer-Hastings, and R. Kreuz, "AutoTutor: A simulation of a human tutor," Cognitive Systems Research, vol. 1, no. 1, pp. 35-51, dec 1999.

[8] C. R. Eliot III, "An Intelligent Tutoring System Based Upon Adaptive Simulation," Ph.D. dissertation, 1996.

[9] C. J. Butz, S. Hua, and R. B. Maguire, "Web-based Bayesian intelligent tutoring systems," pp. 221$242,2008$.

[10] K. Chrysafiadi and M. Virvou, "Student Modeling Approaches: A literature review for the last decade," pp. 4715-4729, Sep 2013.

[11] M. D. Bush, "Computer-assisted language learning: From vision to reality?" CALICO Journal, vol. 25 , no. 3, pp. 443-470, 2008. 
[12] I.Padayachee, "Intelligent tutoring systems: Architecture and characteristics," Tech. Rep., 2002.

[13] V. Slavuj, B. Kovacic, and I. Jugo, "Intelligent tutoring systems for language learning," in 2015 38th International Convention on Information and Communication Technology, Electronics and Microelectronics (MIPRO), no. May. IEEE, May 2015, pp. 814-819.

[14] F. Goullier, "Council of Europe Tools for Language Teaching: Common European Framework and Portfolios,” p. 130, 2006. [Online]. Available: https://www.coe.int/t/dg4/linguistic/Source/Goullier Outils EN.pdf

[15] J. Reye, "Student Modelling based on Belief Networks," Tech. Rep., 2004.

[16] O. H. Hamid, F. Alaiwy, and I. O. Hussien, "A Bayesian Network Model for More Natural Intelligent Tutoring Systems," International Journal of Enhanced Research in Science Technology \& Engineering, vol. 4, no. 2, pp. 109-114, 2015.

[17] J. Pearl and J. Pearl, "Chapter 1 - Uncertainty In AI Systems: An Overview," in Probabilistic Reasoning in Intelligent Systems, 1988, pp. 1-28.

[18] M. Mayo and A. Mitrovic, "Optimising ITS behaviour with Bayesian networks and decision theory," Tech. Rep., 2001.

[19] C.Conati, A. Gertner, and K. Vanlehn, "Using Bayesian networks to manage uncertainty in student modeling," User Modelling and User- Adapted Interaction, vol. 12, no. 4, pp. 371-417, 2002.

[20] R.M. Kaplan, H. Trenholm, D. Gitomer, and L. Steinberg, "Generalizable architecture for building intelligent tutoring systems," Proceedings of the Conference on Artificial Intelligence Applications, p. $458,1993$.

[21] R.A. Howard and J. E. Matheson, "Influence Diagrams," Decision Analysis, vol. 2, no. 3, pp. 127$143,2005$.

[22] "Influence Diagrams," in SpringerReference. Berlin/Heidelberg: Springer-Verlag, 2011. [Online]. Available:https://www.bayesfusion.com/influence-diagrams/ http://www.springerreference.com/ index/doi/10.1007/ SpringerReference 62286

[23] P. Karampiperis and D. G. Sampson, “Adaptive Learning Resources Sequencing in Educational Hypermedia Systems," Educational Technology Society, 2005. 